\title{
Artificial Silk Materials with Enhanced Mechanical Properties and Controllable Structures
}

\author{
Yaopeng ZHANG*, Hui PAN, Jie Luo, Lele ZHANG, Zhaobo LI, Xiangyu HuANG, \\ Yuan JIN, Suna FAN, Yichun HANG, Huili SHAO and Xuechao Hu \\ State Key Laboratory for Modification of Chemical Fibers and Polymer Materials, College of Materials Science and \\ Engineering, Donghua University, Shanghai 201620, P. R. China \\ E-mail :zyp@dhu.edu.cn
}

\begin{abstract}
Spider and silkworm produce animal silks exhibiting outstanding mechanical properties by using smart spinning method. In this paper, a biomimetic spinning of spider silk and silkworm silk was studied from various routes. Regenerated silk fibroin (RSF) aqueous solution was firstly dry-spun to artificial fiber in air at room temperature as the animal silks were made naturally. The conformation transition of the silk fibroin was then induced by post-drawing in ethanol aqueous solution. The oriented crystalline and amorphous regions of the silk fibers contribute to the remarkable mechanical properties of the artificial silk, which exceed those of natural silkworm silk. By mimicking the functions of the spinning apparatus of spider and silkworm, ion and protein concentrations in the RSF aqueous solution were adjusted in microfluidic chips with multiple channels. Inspired by the shape and dimensions of the natural spinning apparatus, a microfluidic chip was designed and applied to the studies of aggregation mechanism of silk fibroin in micro-channel. Moreover, the supermolecular structures of silk fibroin were effectively controlled to reinforce dry-spun/electrospun fibers of RSF by mimicking the core-shell structure of natural animal silks, adding silk sericin or carbon nanotube in spinning solutions and changing the collecting method in electrospinning process.
\end{abstract}

Key Words : Silk fibroin, Silk fiber, Dry-spinning, Electro-spinning, Microfluidic

\section{Introduction}

Natural animal silks from spider and silkworm have many remarkable properties. Especially for dragline silk from Nephila clavipes spider, it is much tougher than Kevlar and carbon fiber, and is even one of the toughest fibers in the world.[1] Furthermore, spider and silkworm use an environmentally friendly dry spinning process to produce silks from all aqueous solutions at ambient temperature and pressure.[2] In addition to the outstanding mechanical properties, silkworm silk shows many other good properties, such as biocompatibility, anti-bacterial property, tailorable biodegradability and good oxygen/water vapor permeability.[3] Thus, silk-based materials have attracted considerable attentions from the researchers in the fields of polymer science and tissue engineering.

In order to obtain the artificial animal silks and mimic the lowcarbon green spinning process, wet spinning and electrospinning techniques have been taken to produce artificial silk fibers from regenerated and recombinant silk protein solutions by partially mimicking the natural spinning process.[4] However, the current biomimetic engineering grossly oversimplified the mechanisms by which natural fibers are formed.[5] Based on the understanding of microstructure and spinning mechanism of natural animal silks, the biomimetic spinning of spider silk and silkworm silk was studied in our group from various routes. Structure controllable spinning process and mechanical properties enhanced artificial silks were successfully developed.[4, 6] Firstly, a biomimetic dry-spinning process for regenerated silk fibroin (RSF) aqueous solution was explored as the natural spinning of animal silks. Secondly, a microfluidic dry-spinning chip was designed to mimic the functions of the spinning apparatus of spider and silkworm, such as ion and protein concentrations adjustment in the RSF aqueous solution. Thirdly, inspired by the composition and the core-shell structure of animal silks, the RSF solution was spun with silk sericin (SS) via coaxial or blending dry spinning and electrospinning. The RSF mats were further reinforced by adding other additives, posttreating and preparing aligned electrospun fibers in multiple layers.

\section{Experimental}

According to the method reported previously,[7] cocoons of Bombyx mori were used to prepare the RSF aqueous solution for dry-spinning, microfluidic spinning and electrospinning. The asspun fibers or mats were post-drawn in $80-90$ vol\% ethanol aqueous solution, and then immersed in the solution for certain minutes or hours. A commercial water-soluble SS polypeptide (SSp, 1 2 kDa, Wuxi Smiss Technology Co., China) was used to prepare RSFSSp blend spinning dopes and RSF- SSp coaxial spinning dopes. During the coaxial spinning, RSF and SSp aqueous solutions were used as the core and shell spinning dopes, respectively. Residual SS (RSS, 35 66 kDa) was prepared along with RSF by degumming B. mori cocoons at different conditions, including the $\mathrm{Na}_{2} \mathrm{CO}_{3}$ concentration and the temperature of the degumming bath. The 
RSS was used to prepare RSF-RSS blend spinning dopes.

Based on photolithography process and plasma bonding technology, [7, 8] microfluidic chips of poly(dimethylsiloxane) (PDMS) (Dow Corning Inc., Midland MI) were fabricated. For multilayered microchip, a cellulose dialysis membrane was bonded between two PDMS layers with epoxy resin.

To investigate the structure of the RSF fibers and solutions, synchrotron radiation micro-focus wide angle X-ray diffraction (SR-WAXD) and small angle X-ray scattering (SR-SAXS) were measured at the BL15U1 and BL16B1 beamlines in Shanghai Synchrotron Radiation Facility (SSRF) with a wavelength of $0.07746 \mathrm{~nm}$ and $0.124 \mathrm{~nm}$, respectively. Other measurements such as FTIR, Raman spectroscopy, birefringence and mechanical properties were performed as reported.[9]

\section{Results and discussions}

\subsection{Structure control of artificial silk by biomimicking natural spinning dope and method}

Normally, RSF solution can only be spun using wet spinning or electrospinning. In natural spinning dope of silkworm and spider, calcium ion plays an important role for protein assembling. Thus we added $\mathrm{CaCl}_{2}$ aqueous solution in $20 \mathrm{wt} \% \mathrm{RSF}$ aqueous solution, which was then concentrated to a highly concentrated spinning dope over $50 \mathrm{wt} \%$. It is surprising that the biomimetic dope can be dry-spun only using a capillary with an inner diameter ranging from 100 to $250 \mu \mathrm{m}$ for the first time.

Although the dry-spinning process is similar to those of silkworm and spider, there are still some differences. Firstly, the structures of RSF molecules are different from those of natural fibroin molecules; secondly, the process is not liquid crystalline spinning as silkworm and spider. Therefore, the as-spun fibers have very low orientation degree and low content of $\beta$-sheet conformation.

We then returned to learn how silkworm spins fiber in air. During the spinning process, the silkworm caterpillar moves its head continuously with a weaving manner and bends or stretches its body cyclically. So the thread out of the spinneret of silkworm can be drawn extensively. To improve the mechanical properties

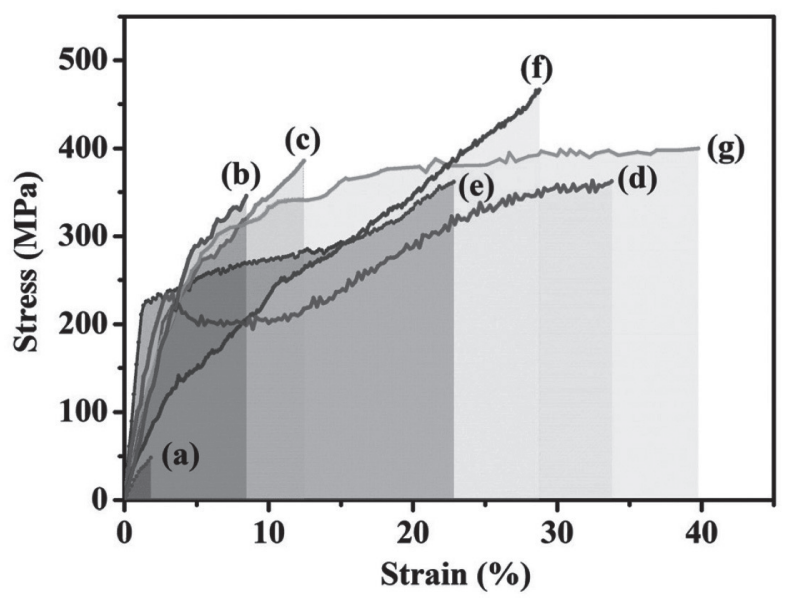

Figure 1 Stress-strain curves of different SF-SS composite fibers, (a) asspun RSF fiber, (b) totally degummed silk, (c) natural silk from $B$. mori's cocoons, (d) post-treated RSF fiber, (e) post-treated RSFSSp coaxial fiber, (f) post-treated RSF-SSp blended fiber, and (g) post-treated RSF-RSS blended fiber. All the draw ratios of the post-treated fibers were 4 .

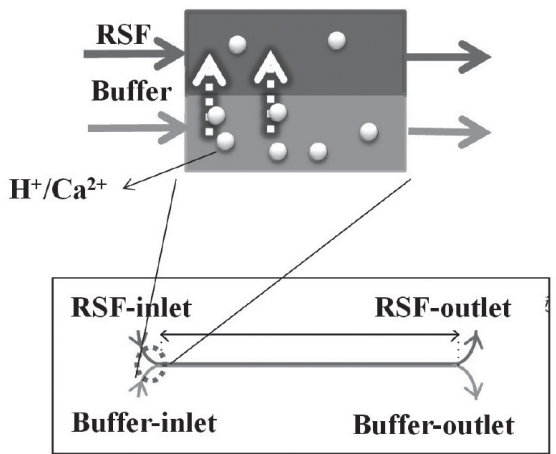

Figure 2 Schematic of the microfluidic chip used for $\mathrm{pH}$ and $\mathrm{Ca}^{2+}$ adjustment (channel size: $500 \mu \mathrm{m}$ in width, $100 \mu \mathrm{m}$ in depth)

of the artificial RSF fibers, it is necessary to post-draw the asspun fibers. Since alcohol is an effective agent to promote the conformational transition of silk fibroin from $\alpha$-helix/random coil to $\beta$-sheet, ethanol aqueous solution $(80 \mathrm{vol} \%$ ) was adopted as a post-treatment agent.[10] A post-drawing device specially designed for ultra-fine monofilament was used for the single-step and multi-step post drawing. Raman, FTIR and WAXD revealed that the post-treatment increased the $\beta$-sheet content, crystallinity and orientation.[3,9] As a result, the mechanical properties of post-treated RSF fiber (Figure 1d) were greatly enhanced and approached to those of natural silkworm silk (Figure $1 \mathrm{~b}$ and $\mathrm{c}$ ). To prepare high-performance silk, it is crucial to understand the relationship between the structures and the mechanical properties of the fibers. SR-WAXD was used to obtain the details of the crystalline structure of dry-spun RSF single fibers. As the posttreatment process was performed, the peak of $(021)(d$-spacing $0.37 \mathrm{~nm}$ ) lattice plane weakened while the peaks of $(020)(d$-spacing $0.45 \mathrm{~nm})$ and (200) ( $d$-spacing $0.43 \mathrm{~nm}$ ) lattice planes grew intensely and became dominated finally. The crystallinity increased with the increase of draw rate and decrease of draw ratio, while the crystalline size of the RSF fibers all decreased with the increase of draw rate and ratio.[9] The investigation of the structure-property relationships of the RSF fibers provides a wealth of possible new insight into the design of high-performance artificial animal silk.

\subsection{Structure control of artificial silk by biomimicking natural spinning apparatus and dynamical adjustment of spinning dope components}

The development of microfluidic technology inspires its application in spinning and even biomimetic spinning of natural animal silk proteins. Since the diameters of the natural spinning ducts dropped from several hundred micrometers to several micrometers for spiders and silkworms, the spinning systems in vivo can be considered as typical microfluidic devices. Therefore,

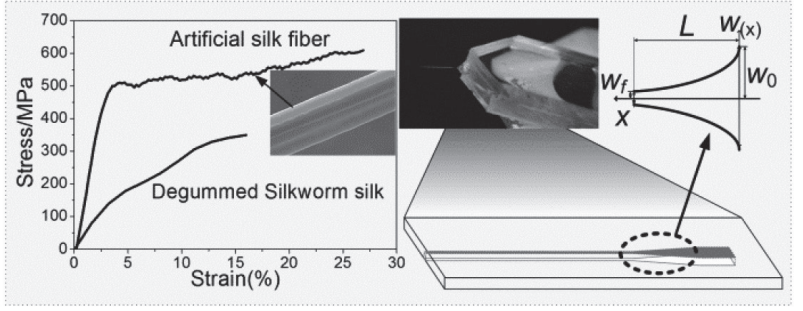

Figure 3 Tough silk fibers were dry-spun from silk fibroin aqueous solution using microfluidic chips with a single channel mimicking the spinning apparatus of spider and silkworm. 
various microfluidic chips were designed to mimic the functions of natural spinning systems, such as $\mathrm{pH} / \mathrm{Ca}^{2+}$ adjustment, fibroin concentration and fiber spinning.[7]

The first chip (Figure 2) was designed and used to dynamically adjust $\mathrm{pH}$ or $\mathrm{Ca}^{2+}$ concentration of RSF solution. Due to the laminar flow characteristic of microfluidics, RSF solution and a buffer $\left(\mathrm{pH}\right.$ or $\left.\mathrm{Ca}^{2+}\right)$ tended to keep their laminar streams without mixing. Thus, the RSF concentration was maintained consequently, while the $\mathrm{pH}$ value or $\mathrm{Ca}^{2+}$ concentration of the RSF solution was dynamically adjusted via ionic diffusion during the RSF solution flowing in the microfluidic channels. The results showed that it was easier to adjust the $\mathrm{pH}$ value of the RSF solution to the target $\mathrm{pH}$ value of the buffer, when the two streams had closer $\mathrm{pH}$ values. In addition, the lower flow rates of the two streams and the decreasing RSF concentration of RSF solution improved the $\mathrm{pH}$ value and $\mathrm{Ca}^{2+}$ concentration dynamically adjustment of the RSF solution.

By biomimicking the dynamical enrichment of the spinning process in vivo, two-layer microfluidic concentrators were designed and used for the enrichment of RSF solution by microdialysis. [11] The microfluidic devices based on regenerated cellulose membrane involved a flow of RSF solution on a donor side and a flow of polyethylene glycol (PEG) aqueous solution on an acceptor side. The results showed that the chips could be used as microconcentrators for RSF solution enrichment. The RSF concentration of the solution could be enriched up to $31.2 \mathrm{wt} \%$ from $12 \mathrm{wt} \%$ by using the microfluidic chip. The enrichment efficiency increased with the increasing acceptor concentration, increasing channel length, depth and decreasing flow rate of RSF solution.

In addition to the mimic of content adjustment of RSF solution, the dimension of spinning apparatus in vivo was also biomimicked in microfluidic chips with a single channel. Raman spectroscopy and SR-SAXS technique were adopted to analyze the conformation transition of RSF and the shape parameters of RSF aggregates in RSF aqueous solutions before and after flowing in the microchannel. Results indicated that the increasing shear time and elongation rate had similar effects on the structure transition of RSF in aqueous solution. When the elongation rate and the shearing time in the microchannels were respectively varied, a proper increase of $\beta$-sheet structure of RSF in its solution $\left(40 \mathrm{wt} \%, \mathrm{pH} 4.8, \mathrm{c}\left(\mathrm{Ca}^{2+}\right)=0.3 \mathrm{M}\right)$ was observed with the increasing elongation rate or shear time in the microchannels.

Based on the above studies, the microfluidic chips were also used to spin RSF fibers from highly concentrated aqueous solution $\left(50 \mathrm{wt} \%, \mathrm{pH} 4.8, \mathrm{c}\left(\mathrm{Ca}^{2+}\right)=0.3 \mathrm{M}\right)$. The microfluidic channels with various geometries as exponential function were adopted to mimic the natural elongation and shearing condition (Figure 3). It was found that the conformation transition of SF and the crystalline structure in RSF as-spun fibers were improved, when the elongation rate, the elongation time, and the shearing rate increased. Thus, the mechanical properties of the as-spun RSF fibers were enhanced. To improve the structure and mechanical properties of the RSF fibers, they were further post-treated. It was obvious that the posttreatment played a significant role in the formation of $\beta$-sheet structure and the improvement of silk II crystalline structure. The post-treated RSF fibers showed excellent mechanical properties with a Young's modulus of $19 \mathrm{GPa}$, a breaking stress of $614 \mathrm{MPa}$, and a breaking strain of $27 \%$.

\subsection{Structure control of artificial silk by biomimicking the components and core-shell structure of natural silk}

Silkworm silk has a core-shell structure and consists of two SF brins which are conglutinated by SS binder. It is revealed that SS avails to the structural transition of SF and shows good biocompatibility and some biological activities.

In order to mimic the composition of silkworm silk, regenerated RSF/SSp blend aqueous solutions were prepared for electrospinning.[12] It was found that the addition of SS in the SF solution could increase the apparent viscosity and spinnability of the solution. The analysis on the structure and properties of the regenerated RSF/SSp mats revealed that the presence of SS facilitated the conformational transition of RSF from random coil/ $\alpha$-helix structure to $\beta$-sheet structure. Consequently, SS played an important role in the improvement of thermal and mechanical properties of the resultant RSF/SSp fibers.

To further mimic the core-shell structure of silkworm silk, the ultra-fine fibers with a core (RSF)-shell (SSp) structure were prepared by coaxial electrospinning using the RSF and SSp aqueous solution as the core and shell spinning dope, respectively (Figure 4).[13] Compared with the electrospun pure SF fibers, the coaxial SF/SS fibers had more stable structure with more $\beta$-sheet (and related intermediate) conformation and accordingly better thermostability and mechanical properties. Therefore, it is hypothesized that besides serving as an adhesive to bind two SF monofilaments together in cocoon, SS may also induce the conformational transition of SF to $\beta$-sheet structure.

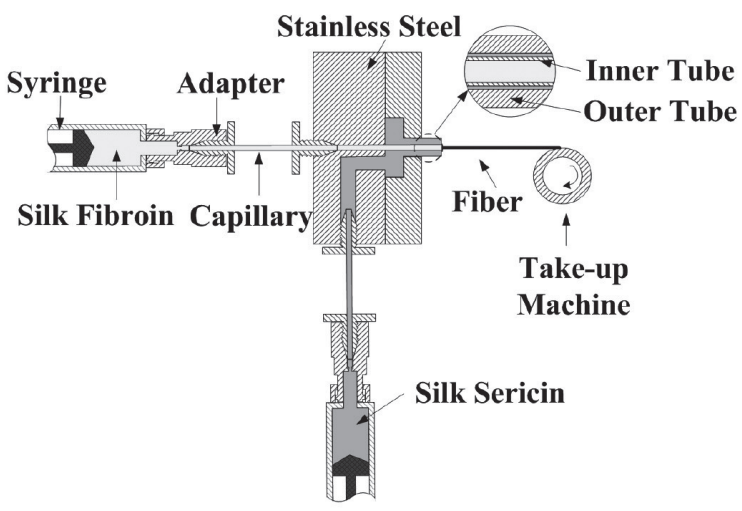

Figure 4 Schematic illustration of coaxial dry-spinning and electrospinning.

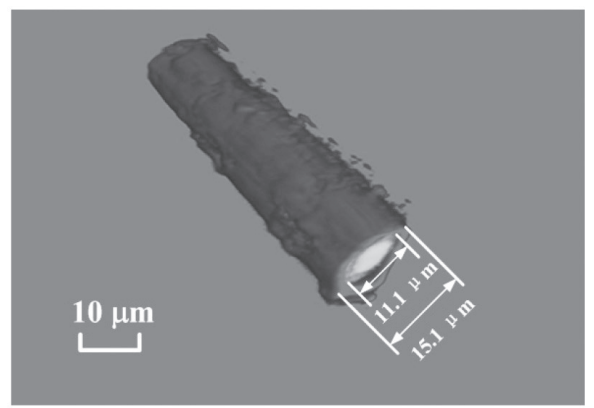

Figure 5 3D image via $\mathrm{z}$-axis serial optical sections scanning of laser scanning confocal microscope (LSCM) for an as-spun RSF-SSp coaxial fiber. Rhodamine $\mathrm{B}$ and calcein aqueous solutions, with the same concentration of $5 \mathrm{mg} / \mathrm{L}$, were respectively pre-added into the shell dopes and core dopes at a ratio of $1: 100(\mathrm{v} / \mathrm{v})$ as fluorescent dyes. 
In addition to the electrospinning, dry-spinning was also adopted for RSF-SS blend and coaxial spinning. The resultant SF/SS fiber exhibited a thick diameter and a clear core-shell structure (Figure 5). It can be seen from Figure 1 that all the post-treated SF/SS fibers showed evident superiority in the mechanical properties compared with the silkworm silk. According to different spinning methods or types of SS, the mechanical properties of the RSF-SS composite artificial silk are tunable. For the post-treated RSF-RSS blended fiber (Figure 1g), it had the highest breaking energy of 60 $\mathrm{kJ} / \mathrm{kg}$, which is much higher than that of natural silk (Figure 1c). The post-treated RSF-SSp blended fiber (Figure 1f) exhibited an impressive breaking stress of $460 \mathrm{MPa}$, but a small initial modulus. However, the post-treated RSF-SSp coaxial fiber (Figure 1e) showed a very inspiring initial modulus level of $18 \mathrm{GPa}$, which is twice higher than that of the post-treated RSF-SSp blend fiber and could rival the stiffness of a forcibly reeled silk and even a spider dragline silk according to some relevant data reported.[2, 14] The high modulus probably resulted from the stiff SSp shell around RSF core(Figure 5). The above results indicate that the fabrication of high-performance silks can be realized through a bio-inspired artificial spinning and a proper post-treatment.

\subsection{Reinforcement of artificial silk mats}

It is well known that the poor mechanical properties of electrospun RSF mats limit their extensive applications. Researchers have tried to reinforce the mats through blending or post-treatment. However, most reinforcement was not effective enough, since only alcohol immersion or post-drawing was applied independently in the post-treatment process. Due to dispersion problem of carbon nanotubes (CNTs) in RSF solution, it was also difficult to prepare composite RSF mats with enhanced mechanical properties. Moreover, the randomly oriented fibers in the typical electrospun SF mats limit the mechanical properties and applications of the mats.

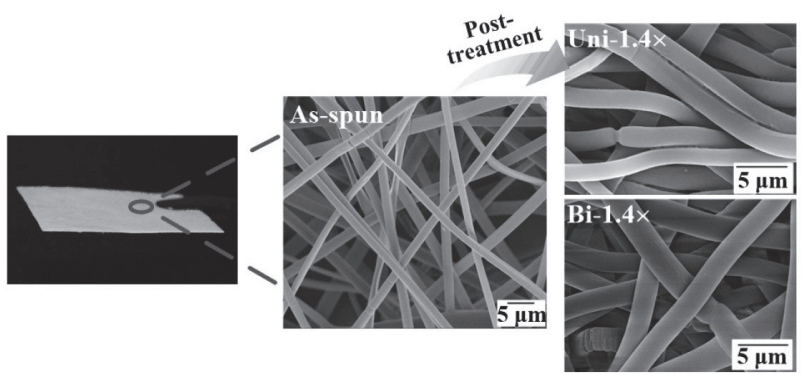

Figure 6 Morphology of RSF scaffolds before and after being uniaxially or biaxially postdrawn in $90 \mathrm{vol} \%$ ethanol solution.

Three strategies were used in our works to reinforce the mats. The first strategy of post-treatment was inspired from the above post-treatment method for RSF dry-spun fiber. A post-drawing method in ethanol aqueous solution was applied to reinforce the RSF scaffolds electrospun from aqueous solution. Draw rate and draw ratio vary during the post-treatment which allows us to accurately control the mechanical properties, thermal stability and degradability. The uniaxial drawing can increase the alignment of fibers parallel to the drawing direction. The RSF scaffolds with uniform arrangement can be acquired by biaxial drawing (Figure 6). Both uniaxial and biaxial drawing can improve the crystallinity of scaffolds, which increased with the increase of draw ratio. The
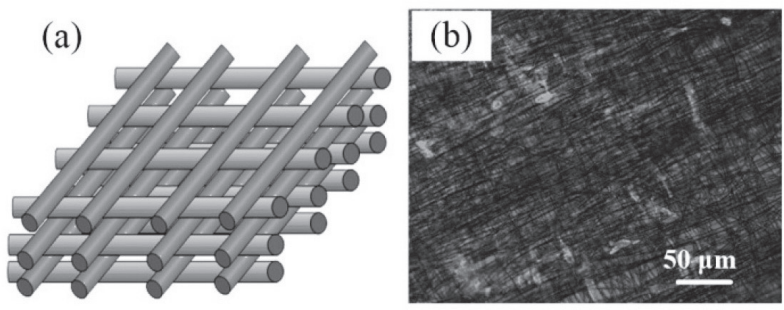

Figure 7 (a) 3D schematic diagram of multi-layered SF mats with a compositing angle of $90^{\circ}$. (b) LSCM images of PIEC cultured for 3 days on electrospun SF mats with aligned fibers.

breaking strength and breaking energy of the post-treated mats at $1.4 \times$ draw ratio and $0.1 \mathrm{~mm} / \mathrm{s}$ draw rate were $8.6 \mathrm{MPa}$ and $172.2 \mathrm{~J} /$ $\mathrm{kg}$, respectively. However, those of the as-spun mats were only 1.8 MPa and 93.2 J/kg, respectively.[3] This can be attributed to the increase of crystallinity and the number of aligned fibers.

The second strategy is blending reinforce agent into spinning dope. Functionalized multiwalled carbon nanotubes (F-MWNTs) were well dispersed in the RSF solutions. The mechanical properties of the RSF electrospun fibers were improved drastically by incorporating F-MWNTs. Compared with the pure RSF electrospun fibers, the composite fibers with $1.0 \mathrm{wt} \%$ F-MWNTs exhibited a 2.8 -fold increase in breaking strength (from 0.84 to $3.24 \mathrm{MPa}$ ), and a 2.1-fold increase in breaking energy(from 9.5 to $29.3 \mathrm{~J} / \mathrm{kg}$ ). [15]

The third strategy is to prepare RSF mats with well-aligned fibers. A multiple layered structure with fibers in different layers oriented in different directions and stacked layer-by-layer is fabricated through rotating the substrate (Figure 7a). Post-treatment methods of ethanol immersion, uniaxial and biaxial stretching, water vapor annealing were carried out to further improve the mechanical performance of the SF mats. By combining ethanol immersion and uniaxial stretching, SF mats had an excellent breaking strength of $10.8 \mathrm{MPa}$ and a high breaking energy of $49.7 \mathrm{~J} / \mathrm{kg}$ along the direction of stretching. From suture retention strength testing, all SF mats had a breaking force higher than $0.8 \mathrm{~N}$ which met the mechanical requirement of tissue engineering scaffolds. Compared with the mats with randomly aligned fibers, the scaffolds with well-aligned fibers are suitable to guide the cell growth with desired anisotropy (Figure 7b). Results demonstrated that porcine iliac endothelial cells (PIECs) could grow in the interior of the SF mats.

\section{Conclusions}

Artificial silk materials were prepared with enhanced mechanical properties and controllable structures from various routes. Inspired of natural animals' spinning process, a smart dry-spinning method was used to prepare artificial silk. Microfluidic technology was employed for animal apparatus imitation. Furthermore, artificial silk with core-shell structure was successfully produced based on the former techniques. The post-treatment with the controllable spinning parameters regulated the structures of artificial silks which mechanical properties were enhanced approaching or exceeding that of natural cocoon silk. The biomaterials prepared from RSF with enhanced mechanical properties and controllable structures have been proved to be good candidates for tissue engineering.

Acknowledgement - This work is supported by the National

Natural Science Foundation of China (50803011, 21274018, 
81170641), Specialized Research Fund for the Doctoral Program of Higher Education (200802550001), the Shanghai RisingStar Program (12QA1400100), Innovation Program of Shanghai Please replace page 5, line 4 to "Municipal Education Commission (12ZZ065), DHU Distinguished Young Professor Program (A201302) and the Fundamental" Research Funds for the Central Universities. We also would like to thank Dr. W. Wei, Ms. N.N. Jiang, Mr. M. J. Sun, Ms. Y. Huang, and Mr. Y.Y. Li for their contribution to this work.

\section{References}

[1] Vollrath, F., Knight, D. P.; "Liquid crystalline spinning of spider silk", Nature, 410(6828), 541-548. (2001).

[2] Shao, Z. Z., Vollrath, F.; "Materials: Surprising strength of silkworm silk", Nature, 418(6899), 741-741. (2002).

[3] Fan, S. N., Zhang, Y. P., Shao, H. L., Hu, X. C.; "Electrospun regenerated silk fibroin mats with enhanced mechanical properties", Int. J Biol. Macromol., 56, 83-88. (2013).

[4] Wei, W., Zhang, Y. P., Zhao, Y. M., Luo, J., Shao, H. L., Hu, X. C.; "Bio-inspired capillary dry spinning of regenerated silk fibroin aqueous solution", Mater. Sci. Eng. C, 31(7), 16021608. (2011).

[5] Luo, J., Zhang, Y. P., Huang, Y., Ye, Y., Shao, H. L., Hu, X. C.; "A microfluidic chip for biomimicking the spinning duct of silk worm", Proceedings of 2009 International Conference on Advanced Fibers and Polymer Materials, 1\&2, 960-962. (2009).

[6] Wei, W., Zhang, Y. P., Zhao, Y. M., Shao, H. L., Hu, X. C.; "Dry spinning of regenerated silk fibroin aqueous solution", Proceedings of 2009 International Conference on Advanced Fibers and Polymer Materials, 1\&2, 762-764. (2009).

[7] Luo, J., Zhang, Y. P., Huang, Y., Shao, H. L., Hu, X. C.; "A bio-inspired microfluidic concentrator for regenerated silk fibroin solution", Sensor Actuat. B, 162(1), 435-440. (2012).

[8] Mcdonald, J. C., Chabinyc, M. L., Metallo, S. J., Anderson, J. R., Stroock, A. D., Whitesides, G. M.; "Prototyping of microfluidic devices in poly(dimethylsiloxane) using solidobject printing", Anal. Chem., 74(7), 1537-1545. (2002).

[9] Sun, M. J., Zhang, Y. P., Zhao, Y. M., Shao, H. L., Hu, X. C.; "The structure-property relationships of artificial silk fabricated by dry-spinning process", J Mater. Chem., 22(35), 18372-18379. (2012).

[10] Wei, W., Zhang, Y. P., Shao, H. L., Hu, X. C.; "Posttreatment of the dry-spun fibers obtained from regenerated silk fibroin aqueous solution in ethanol aqueous solution", J Mater. Res., 26(9), 1100-1106. (2011).

[11] Kim, C. J., Ryu, C. G., Kim, L. W., Sim, S. J., Chae, H. Y.; "Microfluidic dialysis device fabrication for protein solution enrichment and its enrichment enhancement by plasma surface treatment of a membrane", J Korean Phys. Soc., 51(3), 993-999. (2007).

[12] Hang, Y. C., Zhang, Y. P., Jin, Y., Shao, H. L., Hu, X. C.; "Preparation and characterization of electrospun silk fibroin/ sericin blend fibers", J Mater. Res., 26(23), 2931-2937. (2011).

[13] Hang, Y. C., Zhang, Y. P., Jin, Y., Shao, H. L., Hu, X. C.; "Preparation of regenerated silk fibroin/silk sericin fibers by coaxial electrospinning", Int. J Biol. Macromol., 51(5), 980986. (2012).

[14] Fu, C. J., Shao, Z. Z., Fritz, V.; "Animal silks: their structures, properties and artificial production", Chem. Commun., (43), 6515-6529. (2009).

[15] Pan, H., Zhang, Y. P., Hang, Y. C., Shao, H. L., Hu, X. C., Xu, Y. M., Feng, C.; "Significantly Reinforced Composite Fibers Electrospun from Silk Fibroin/Carbon Nanotube Aqueous Solutions", Biomacromolecules, 13(9), 2859-2867. (2012). 